\title{
Graph Color Extensions: When Hadwiger's Conjecture and Embeddings Help
}

\author{
Michael O. Albertson \\ Department of Mathematics \\ Smith College, Northampton, MA 01063 USA \\ albertson@smith.edu \\ Joan P. Hutchinson \\ Department of Mathematics and Computer Science \\ Macalester College, St. Paul, MN 55105 USA \\ hutchinson@macalester.edu
}

Submitted: April 29, 2002; Accepted: September 12, 2002.

MR Subject Classifications: 05C15, 05C10

\begin{abstract}
Suppose $G$ is $r$-colorable and $P \subseteq V(G)$ is such that the components of $G[P]$ are far apart. We show that any $(r+s)$-coloring of $G[P]$ in which each component is $s$-colored extends to an $(r+s)$-coloring of $G$. If $G$ does not contract to $K_{5}$ or is planar and $s \geq 2$, then any $(r+s-1)$-coloring of $P$ in which each component is $s$-colored extends to an $(r+s-1)$-coloring of $G$. This result uses the Four Color Theorem and its equivalence to Hadwiger's Conjecture for $k=5$. For $s=2$ this provides an affirmative answer to a question of Thomassen. Similar results hold for coloring arbitrary graphs embedded in both orientable and non-orientable surfaces.
\end{abstract}

\section{Introduction}

Suppose $G$ is an $r$-colorable graph and $P \subseteq V(=V(G))$. One naturally asks if an $r$ coloring of $G[P]$ extends to a $t$-coloring of $G$ where $r \leq t$. This question is $N P$-complete even with severe restrictions on $G$. Its complexity has been well studied; for a survey see [24].

Several years ago Thomassen asked if a planarity assumption and a distance constraint on $P$ would help.

Question 1 [21] Suppose $G$ is a planar graph and $P \subseteq V$ is such that the distance between any two vertices in $P$ is at least 100. Can a 5-coloring of $P$ be extended to a 5-coloring of $G$ ? 
The answer to Thomassen's question is yes, but the result does not require any topology.

Theorem 1 [1] If $\chi(G)=r$ and the distance between any two vertices in $P$ is at least 4 , then any $(r+1)$-coloring of $P$ extends to an $(r+1)$-coloring of all of $G$.

If $\chi(G)=r$ there is no similar extension theorem with $r$ colors even if $G$ is planar and $r=4$ [1]. In response to Theorem 1 Thomassen asked about extending colorings of bipartite subgraphs. If $P \subseteq V$ let $G[P]$ denote the subgraph of $G$ induced by $P$.

Question 2 (Problem 1 in [22]) Suppose $G$ is a planar graph, $G[P]$ is bipartite, and any two components of $G[P]$ have distance at least 100 from each other. Can any 5-coloring of $G[P]$ in which each component is 2-colored be extended to a 5 -coloring of $G$ ?

In this paper we show that the answer to Thomassen's second question is also yes. Although the embedding assumption is partially needed, a weaker assumption on minors is the essential ingredient. Besides considering extensions of 2-colored subgraphs we investigate when colorings of more general induced subgraphs of $G$ extend to colorings of all of $G$. We also obtain some results for embedded graphs.

Suppose $P=P_{1} \cup P_{2} \cup \cdots \cup P_{k} \subseteq V$ where $P_{1}, P_{2}, \cdots, P_{k}$ induce the connected components of $G[P]$. Throughout this paper we will assume that $\chi(G) \leq r$ and $\chi\left(G\left[P_{i}\right]\right) \leq$ $s$. We say that $d(P) \geq \rho$ if for every pair of vertices $x \in P_{i}, y \in P_{j}(i \neq j)$ the distance in the graph (i.e., the number of edges in a shortest path) between $x$ and $y$ is at least $\rho$. If $S \subseteq V, N(S)$ denotes the set of vertices that are not in $S$ but are adjacent to at least one vertex in $S$. Inductively $N^{i}(S)$ denotes the set of vertices that are not in $N^{i-1}(S)$ but are adjacent to at least one vertex in $N^{i-1}(S)$. So $N^{i}(S)$ consists of those vertices in $G$ whose distance from $S$ is exactly $i$.

In Section 2 we show that if $d(P) \geq 4$, then any $(r+s)$-coloring of $G[P]$ in which each $G\left[P_{i}\right]$ is $s$-colored (not necessarily with the same colors) extends to an $(r+s)$-coloring of $G$. This is best possible both with respect to the distance constraint and the number of colors needed. This result contrasts with the result below, an earlier extension theorem in which each precolored component is an $r$-clique.

Theorem 2 [7] If $\chi(G)=r$ and $G[P]$ consists of copies of $K_{r}$ whose pairwise distance is at least $3 r$, then any $(r+1)$-coloring of $G[P]$ extends to an $(r+1)$-coloring of all of $G$.

Our original Section 3 considered planar graphs. Carsten Thomassen insightfully noted that our proofs yielded more, namely that graphs containing no $K_{5}$ minor played the key role here [23] . A graph $G$ is said to contain a graph $H$ as a minor if some sequence of edge deletions, vertex deletions, and edge contractions transforms $G$ into $H$. Since deletion and contraction preserve planarity (and embeddability in any fixed surface), a planar graph cannot contain a $K_{5}$ minor since $K_{5}$ is not planar. However, being $K_{5}$-minor free is not enough to imply planarity since a graph is planar if and only if it contains no $K_{5}$ or $K_{3,3}$ minor [25]. Minors that are complete graphs are believed to have important implications for the chromatic number; the following is one of the outstanding conjectures of graph theory. 
Conjecture 1 Hadwiger's Conjecture [15] If a graph $G$ is $K_{k}$-minor free, then it can be $(k-1)$-colored.

This conjecture is known to hold for $k \leq 6$ : see [17] for the history and current status of the problem. The case of $k=5$ was shown to be equivalent to the Four Color Theorem [25] and so holds [9, 19]. More recently Robertson, Seymour and Thomas [20] have demonstrated the case of $k=6$ using their work on the Four Color Theorem and beyond [19].

In Section 3 we show that if $G$ is $K_{5}$-minor free, $d(P) \geq 8$, and $s \geq 2$, then any $(r+s-1)$-coloring of $G[P]$ in which each $G\left[P_{i}\right]$ is $s$-colored (again not necessarily with the same colors) extends to an $(r+s-1)$-coloring of $G$; the same result holds for $G$ planar. Thus, when extending colorings of $K_{5}$-minor free and planar graphs with $s \geq 2$, we need one fewer color than the results of Section 2 suggest. This doesn't happen when $s=1$. Although our proofs fail if $d(P) \leq 7$, we do not know what the right distance hypothesis is.

Section 4 looks at graphs embedded in a given surface. Here if one is extending a precoloring using (nearly) the Heawood number of colors, then the total number of colors required is one fewer than might be anticipated from Section 2. Although these results are similar to the extension theorems for planar (and $K_{5}$-minor free) graphs, there is one important difference. For planar graphs if $P$ is an independent set, then an extra color is required for an extension theorem. Again for planar graphs when $s \geq 2$ and $G[P]$ is $s$-colored we need $r-1$ additional colors for an extension theorem. For graphs embedded in a surface of positive Euler genus, whenever $s \geq 1$ and $G[P]$ is $s$-colored we need only $r-1$ additional colors.

\section{Extending without Hadwiger or Embeddings}

Theorem 3 Suppose $G$ is $r$-colorable and $P \subseteq V$ induces an $s$-colorable subgraph such that $d(P) \geq 4$. Any $(r+s)$-coloring of $G[P]$ in which each $G\left[P_{i}\right]$ is $s$-colored can be extended to an $(r+s)$-coloring of $G$.

Proof Begin by coloring $G[V-P]$ with the colors $1,2, \cdots, r$. Next for each $i$ color the vertices of $G\left[P_{i}\right]$ with the colors $r+1, r+2, \cdots, r+s$ subject to the following two conditions. First, the color classes in this coloring are identical with the color classes in the precoloring. Second, if the precoloring assigns a particular color class of vertices of $G\left[P_{i}\right]$ a color from $r+1, r+2, \cdots, r+s$, then this coloring uses the same color on that color class. At the moment we have an $(r+s)$-coloring of $G$ in which the colors $r+1, r+2, \cdots, r+s$ are only used on vertices in $G[P]$. Suppose a color class in $G\left[P_{i}\right]$ is colored with the color $r+j$ when the precoloring has it assigned color $m$. We simultaneously perform a Kempe change on every vertex in this color class changing its color from $r+j$ to $m$. If $v$ is a vertex in $G$ that was colored $m$ and was adjacent to $w$ in $G\left[P_{i}\right]$ that had its color changed, then $v$ will now be colored $r+j$. The Kempe chains stop after at most one stage since no vertex in $N^{2}\left(P_{i}\right)$ can be colored $r+j$. Thus all of these changes are confined to the vertices 
of $P \cup N(P)$. Since $d(P) \geq 4$ no vertices that have their colors changed from different components of $P$ can be adjacent. Thus we now have a coloring of $G$ that extends the desired precoloring of $G[P]$.

The distance constraint and the total number of colors needed is best possible when $s=1[1,7]$. Below we see that for $s \geq 2$ the total number of colors needed is also best possible.

Example 1 For ease of presentation we assume that $t=\frac{r}{s}$ is an integer. Our graph contains $2 r$ vertices. The non-precolored part will be an $r$-clique labelled $v_{1}, v_{2}, \cdots, v_{r}$. The precolored part will have just one component. We construct $G[P]$ by taking $t$ copies of $K_{s}$. For $1 \leq i \leq t$ we will label these vertices $u_{i, 1}, u_{i, 2}, \cdots, u_{i, s}$. For $1 \leq i \leq t-1$, we add edges joining $u_{i, s}$ with $u_{i+1,1}$. This makes a path of the $t$ copies of $K_{s}$ ensuring that $G[P]$ is connected. Imagine a perfect matching, say $M$, by joining $u_{i, j}$ with $v_{(i-1) s+j}$. Now both $G[P]$ and $G[V-P]$ have exactly $r$ vertices. Between these two pieces add all edges from a $K_{r, r}$ except for those in the matching $M$. The resulting graph $G$ will be our example. First $\chi(G)=r$ since each vertex in $G[P]$ can be assigned the color of the vertex in the $K_{r}$ that it is matched with in $M$. Second if the vertices in $P$ are $s$-colored by assigning the color $j$ to the vertex $u_{i, j}$, then none of the colors $1,2, \cdots, s$ can be used on any of the vertices of the $K_{r}$ so a total of $r+s$ colors will be needed to finish the coloring. If $s \nmid r$, a similar construction using a total of $\frac{2 r \cdot s}{\operatorname{gcd}(r, s)}$ vertices works.

The preceding example with $s=2$ and $r=4$ differs by just one edge from an example originally presented in [1]. This graph was used there to illuminate Question 2 - in particular to show that without the assumption of planarity the answer would be no. Note that the issue in this example is not a conflict caused by the precoloring of different components. It is rather the difficulty of extending the precoloring of one component to its first neighborhood.

\section{$3 \quad K_{5}$-minor free and Planar Graphs}

Theorem 4 Suppose $G$ does not contain $K_{5}$ (respectively, $K_{6}$ ) as a minor, $P=P_{1} \cup P_{2} \cup$ $\cdots \cup P_{k} \subseteq V, G[P]$ is bipartite, and $d(P) \geq 8$. Any 5-coloring (resp., 6-coloring) of $G[P]$ in which each component is 2-colored can be extended to a 5-coloring (resp., 6-coloring) of $G$.

Proof Suppose $G$ does not contain $K_{5}$ as a minor. For all $1 \leq i \leq k$ perform the following two steps. First, remove edges from $G\left[P_{i}\right]$ until it becomes a tree. Note that removing these edges does not alter the color classes in any 2-coloring of each component tree. Second, contract all of the remaining edges in $G\left[P_{i}\right]$ to obtain the new vertex $v_{i}$ and erase multiple edges. Call this graph $G^{\prime}$. Note that $G^{\prime}$ does not contract to $K_{5}$ and so is 4-colorable by (the proven case of) Hadwiger's Conjecture with $k=5$. We take a 4-coloring of $G^{\prime}$, say $c$, using the colors $\{1,2,3,4\}$ and transfer it to $G[V-P]$. We now need to color the vertices of $G[P]$. We will proceed one component at a time. Suppose 
$c\left(v_{i}\right)=1$. This means that no first neighbor of $G\left[P_{i}\right]$ is colored 1 . So we arbitrarily assign the colors 1 and 5 to the two color classes of $G\left[P_{i}\right]$. If the precoloring happened to assign these two classes the colors 1 and 5 we would be done.

For the moment suppose these two color classes are assigned the colors 2 and 3 in the precoloring. First perform a $(3,5)$-Kempe change at every vertex $v$ in $G\left[P_{i}\right]$ that is colored 5. Now every vertex in $G\left[P_{i}\right]$ that is supposed to be colored 3 is. (Note that if the precoloring had given these two classes the colors 1 and 3, we would be done right now.) Second perform a $(1,5)$-Kempe change at every vertex in $G\left[P_{i}\right]$ that is colored 1. Finally perform a $(2,5)$-Kempe change at every vertex in $G\left[P_{i}\right]$ that is currently colored 5 . These three Kempe changes have the effect of making the colors on $G\left[P_{i}\right]$ agree with those of the precoloring. Furthermore these color changes are confined to $P_{i} \cup N\left(P_{i}\right) \cup N^{2}\left(P_{i}\right) \cup N^{3}\left(P_{i}\right)$.

The remaining case would be if the precoloring had assigned the colors 2 and 5 to these two color classes of vertices. The natural temptation, to switch colors 1 and 2 might send this change throughout the graph. Here we assign the color 5 to the vertices needing 2 and the color 1 to the vertices needing 5. Then we perform a $(2,5)$-Kempe change on $G\left[P_{i}\right]$ followed by a $(1,5)$-Kempe change. Here the color changes are confined to $P_{i} \cup N\left(P_{i}\right) \cup N^{2}\left(P_{i}\right)$.

Since $d(P) \geq 8$, in every case the color changes for the separate components do not affect any pair of adjacent vertices. Thus we have transformed the original 4-coloring of $G^{\prime}$ into a 5-coloring of $G$ that agrees with the precoloring on each $G\left[P_{i}\right]$.

When $G$ does not contain $K_{6}$ as a minor, Hadwiger's Conjecture, proven also for $k=6$, shows that the contracted graph $G^{\prime}$ can be 5-colored and the same proof yields a 6-color extension theorem

The preceding theorem extends to precolorings in which each precolored component is $s$-colored.

Theorem 5 Suppose $G$ does not contain $K_{5}$ (respectively, $K_{6}$ ) as a minor, $P=P_{1} \cup$ $P_{2} \cup \cdots \cup P_{k} \subseteq V, G[P]$ is $s$-colorable where $s \geq 3$, and $d(P) \geq 8$. Any $(3+s)$-coloring (resp., $(4+s)$-coloring) of $G[P]$ in which each component is $s$-colored can be extended to a $(3+s)$-coloring (resp., $(4+s)$-coloring) of $G$.

Proof Construct $G^{\prime}$ as in the proof of Theorem 4. Assume that $G^{\prime}$ is 4-colored using colors $1,2,3,4$ and transfer the coloring of $G^{\prime}$ to a coloring of $G[V-P]$. Complete this to an $(s+$ $3)$-coloring of $G$ one component at a time. Suppose $c\left(v_{i}\right)=1$. Use the colors $1,5,6, \ldots, s+3$ on the vertices of $G\left[P_{i}\right]$ taking care that the color classes in this coloring coincide with the color classes in the $s$-precoloring of $G\left[P_{i}\right]$. For the moment assume that $s=3$. Thus the color classes are currently assigned colors 1,5,6 and the color classes associated with the precoloring of $G\left[P_{i}\right]$ will be one of $\{1,2,3\},\{2,3,4\},\{1,2,5\},\{2,3,5\},\{1,5,6\}$, or $\{2,5,6\}$. Any case in which one color class is already correct can be finished using an argument from the proof of Theorem 4 . This leaves only the case in which the precoloring has $\{2,3,4\}$ on its color classes. In this case we successively make the following Kempe changes, $(4,6),(3,5),(1,5),(2,5)$. Note that every Kempe change uses a color that does not originally occur in $G[V-P]$. Each such change might alter the color on a vertex that 
is adjacent to a previously altered vertex. Even though there are four Kempe changes, they don't use any color more than three times. Thus the changes are confined to $P_{i} \cup$ $N\left(P_{i}\right) \cup N^{2}\left(P_{i}\right) \cup N^{3}\left(P_{i}\right)$. When $s>3$ we note that we may assume that there are at most three color classes in the precoloring of $G[P]$ that are not correctly colored by our coloring of $G^{\prime}$. Thus the proof above works then as well. When $G$ is $K_{6}$-minor free, the argument is essentially the same.

Corollary 5.1 Suppose $G$ is planar, $P=P_{1} \cup P_{2} \cup \cdots \cup P_{k} \subseteq V, G[P]$ is $s$-colorable where $s \geq 2$, and $d(P) \geq 8$. Any $(3+s)$-coloring of $G[P]$ in which each component is $s$-colored can be extended to a $(3+s)$-coloring of $G$.

Note that this corollary with $s=2$ answers Question 2 of Thomassen in the affirmative. We do not know if the theorems in this section are best possible with respect to the distance constraints. As we show below they are best possible with respect to the number of colors needed.

Example 2 Begin with a copy of $K_{3}$ whose vertices are labelled $u, v, w$. Next let $G[P]$ be a path with vertices $u_{1}, \cdots, u_{s}, v_{1}, \cdots, v_{s}, w_{1}, \cdots, w_{s}$. Suppose $u$ is adjacent to $u_{1}, u_{2}, \cdots, u_{s}$, $v$ is adjacent to $v_{1}, \cdots, v_{s}$ and $w$ is adjacent to $w_{1}, \cdots, w_{s}$. Suppose each vertex in $G[P]$ is precolored with its subscript. $G$ is planar and so does not contract to $K_{5}$, and $s+3$ colors are both necessary and sufficient to extend the coloring of $G[P]$ to all of $G$. The same construction, beginning with a copy of $K_{4}$, creates a graph that does not contract to $K_{6}$ and for which $s+4$ colors are necessary and sufficient to extend the coloring of $G[P]$ to all of $G$.

\section{Graphs in Surfaces}

In this section we consider extending precolorings of graphs embedded in surfaces of positive Euler genus. If $\epsilon \leq 2$ is the Euler characteristic of a surface, we let $g^{*}=$ $2-\epsilon$ denote the Euler genus. The orientable surface $S_{g}$, the sphere with $g$ handles, has (orientable) genus $g$ and Euler genus $g^{*}=2 g$. The non-orientable surface $N_{k}$, the sphere with $k$ crosscaps, has Euler genus $g^{*}=k$. If $G$ is embedded in $S$, a surface of Euler genus $g^{*}>0$, Heawood showed that $G$ can be colored with $H\left(g^{*}\right)$ colors where $H\left(g^{*}\right)=\left\lfloor\frac{7+\sqrt{24 g^{*}+1}}{2}\right\rfloor[16,18]$. Dirac and Ungar showed that if $G$ is embedded in $S_{g}(g>0)$, then $G$ requires $H\left(g^{*}\right)$ colors if and only if $G$ contains $K_{H\left(g^{*}\right)}$ as a subgraph [10, 13]. The analogous results for $N_{k}$ were derived in $[10,2]$. In these latter results $N_{2}$, the Klein bottle, is an exception. Franklin [14] showed that any graph embedded in $N_{2}$ can be 6-colored and we showed that a 6-chromatic graph embedded in $N_{2}$ need not contain $K_{6}$ [2], but does contract to $K_{6}[4]$. As a consequence Hadwiger's Conjecture holds for $k=H\left(g^{*}\right)$ for graphs in a surface of Euler genus $g^{*}>0$. In addition the case of $k=H\left(g^{*}\right)-1$ is also known to hold except possibly for $g^{*}=3[3,4,11,12]$. Because of the stronger containment results for $k=H\left(g^{*}\right)$, we choose to primarily phrase the results of this 
section in terms of embedding and colorability, rather than using minors. We include the related minor results as a final theorem.

We begin with the case when $P$ is an independent set. This is of interest because, unlike for 4-colorable graphs, there is no need for an additional color.

Theorem 6 Suppose $G$ is embedded in $S$, a surface of Euler genus $g^{*}>0$, and $P \subseteq V$ is an independent set in $G$ such that $d(P) \geq 6$. Any $H\left(g^{*}\right)$-coloring of $G[P]$ extends to an $H\left(g^{*}\right)$-coloring of $G$ except possibly when $S$ is $N_{3}$.

Proof The results for the projective plane $\left(S=N_{1}\right)$ and the torus $\left(S=S_{1}\right)$ are proved in [5]. Any 7-coloring of a graph embedded in $N_{2}$, the Klein bottle, extends by Theorem 1. The case of extending 6-precolorings of graphs embedded in $N_{2}$ is unsettled as is the case of extending 7-precolorings of graphs embedded in $N_{3}$. It is well known that if $\mathrm{G}$ is a graph with $n$ vertices embedded in a surface $S$ of Euler genus $g^{*}$, then the average degree of $G$ is at most $6+\frac{6\left(g^{*}-2\right)}{n}$. It is straightforward to check that if $n \geq 2 H\left(g^{*}\right)$ and $g^{*} \geq 4$, then this average is less than $H\left(g^{*}\right)-1$. Consequently if $n \geq 2 H\left(g^{*}\right)$ we can remove a vertex $x$ such that $\operatorname{deg}(x) \leq H\left(g^{*}\right)-2$. We continue doing this until the number of vertices left in $G$ is less than $2 H\left(g^{*}\right)$. At this time we can color the reduced graph. Since there are fewer than $2 H\left(g^{*}\right)$ vertices we can arrange that the last color will be used either once or not at all. We then insert and color the vertices that we removed from $G$ in the opposite order from which we removed them. Each time we insert a vertex it has at most $H\left(g^{*}\right)-2$ neighbors. Consequently we have at least two color choices so we can safely avoid the last color. Therefore the original graph has an $H\left(g^{*}\right)$-coloring in which the last color is used at most once. If the last color is used not at all we invoke Theorem 1. If the last color is used exactly once we invoke Theorem 1 of [8].

Next we suppose that $G[P]$ is 2-colorable. Unlike the plane (or with $K_{5}$-minor free graphs) there is an increase in the number of colors needed for an extension theorem. In the former cases the jump occurred when precoloring an independent set. Here it occurs when we precolor a bipartite graph.

Theorem 7 Suppose $G$ is embedded in $S$, a surface of Euler genus $g^{*}>0, P=P_{1} \cup$ $P_{2} \cup \cdots \cup P_{k} \subseteq V$, and $d(P) \geq 8$. Any $\left(H\left(g^{*}\right)+1\right)$-coloring of $G[P]$ in which each component is 2-colored can be extended to an $\left(H\left(g^{*}\right)+1\right)$-coloring of $G$. Generally any $\left(H\left(g^{*}\right)+s-1\right)$-coloring of $G[P]$ in which each $G\left[P_{i}\right]$ is $s$-colored can be extended to an $\left(H\left(g^{*}\right)+s-1\right)$-coloring of $G$.

Proof This is the same as the proof of Theorems 4 and 5 since contraction preserves embeddability in a surface.

The preceding is best possible with respect to the number of colors needed, except possibly for the Klein bottle.

Example 3 We begin with an embedding of $K_{H\left(g^{*}\right)}$ in $S$ a surface of Euler genus $g^{*}>$ $0, S \neq N_{2}$. We remove one vertex, say $x$, to have an embedding of $K_{H\left(g^{*}\right)-1}=G[V-P]$. 
Let the vertices be labelled $v_{1}, v_{2}, \cdots, v_{H\left(g^{*}\right)-1}$ in the order in which they border the face that corresponds with where $x$ used to be. Let $G[P]$ consist of a path with $s\left(H\left(g^{*}\right)-1\right)$ vertices, say $u_{1,1}, u_{1,2}, \cdots u_{1, s}, u_{2,1}, \cdots, u_{H\left(g^{*}\right)-1, s}$. This path can be placed in the face that used to hold the vertex $x$. For $1 \leq j \leq\left(H\left(g^{*}\right)-1\right)$ let $v_{j}$ be adjacent to $u_{j, 1}, u_{j, 2}, \cdots, u_{j, s}$. The precoloring of $G[P]$ will assign to each vertex its second subscript as its color. To extend this precoloring we will need the colors $1,2, \cdots, s$ together with $H\left(g^{*}\right)-1$ additional colors for the clique.

Sometimes we can extend with fewer colors.

Theorem 8 Suppose $G$, not containing $K_{H\left(g^{*}\right)-1}$, is embedded in $S$ a surface of Euler genus $g^{*}>0, P=P_{1} \cup P_{2} \cup \cdots \cup P_{k} \subseteq V$, and $d(P) \geq 8$. Any $H\left(g^{*}\right)$-coloring of $G[P]$ in which each component is 2-colored can be extended to an $H\left(g^{*}\right)$-coloring of $G$. Generally any $\left(H\left(g^{*}\right)+s-2\right)$-coloring of $G[P]$ in which each component is $s$-colored where $s \geq 3$ can be extended to an $\left(H\left(g^{*}\right)+s-2\right)$-coloring of $G$.

Proof The proof begins the same way as the proof of Theorem 4. We focus our attention on $G^{\prime}$ - in particular on the largest clique it might contain. By the distance constraint on $P$ any clique in $G^{\prime}$ contains at most one $v_{i}$. Since $G$ did not contain a $K_{H\left(g^{*}\right)-1}, G^{\prime}$ cannot contain a $K_{H\left(g^{*}\right)}$. Consequently $G^{\prime}$ is $\left(H\left(g^{*}\right)-1\right)$-colorable. The proof will then proceed as in the proof of Theorem 4. The generalization follows the proof of Theorem 5 .

Theorem 9 Suppose $G$ is embedded in $S$ a surface of Euler genus $g^{*}>0, G$ does not contract to $K_{H\left(g^{*}\right)}$ (respectively, to $K_{H\left(g^{*}\right)-1}$ and $\left.g^{*} \neq 3\right), P=P_{1} \cup P_{2} \cup \cdots \cup P_{k} \subseteq V$, and $d(P) \geq 8$. Any $H\left(g^{*}\right)$-coloring (resp., $\left(H\left(g^{*}\right)-1\right)$-coloring) of $G[P]$ in which each component is 2-colored can be extended to an $H\left(g^{*}\right)$-coloring (resp., $\left(H\left(g^{*}\right)-1\right)$-coloring) of $G$. Generally any $\left(H\left(g^{*}\right)+s-2\right)$-coloring (resp., $\left(H\left(g^{*}\right)+s-3\right)$-coloring) of $G[P]$ in which each component is $s$-colored where $s \geq 3$ can be extended to an $\left(H\left(g^{*}\right)+s-2\right)$ coloring (resp., $\left(H\left(g^{*}\right)+s-3\right)$-coloring) of $G$.

Proof The proof begins as in the proof for Theorem 8. When $G$ does not contract to $K_{H\left(g^{*}\right)}$ (resp., $K_{H\left(g^{*}\right)-1}$ ), neither does $G^{\prime}$. By Hadwiger's Conjecture, proven for these cases on surfaces, $G^{\prime}$ is $\left(H\left(g^{*}\right)-1\right)$-colorable (resp., $\left(H\left(g^{*}\right)-2\right)$-colorable). The proof then proceeds as for Theorem 8 .

In a subsequent paper [6] we consider additional results on coloring extensions for locally planar graphs.

\section{References}

[1] Michael O. Albertson, You can't paint yourself into a corner, J. Combinatorial Theory, Series B 78 (1998), 189-194.

[2] Michael O. Albertson and Joan P. Hutchinson, The three excluded cases of Dirac's map color theorem, Ann. New York Acad. Sciences 319 (1979), 7-17. 
[3] Michael O. Albertson and Joan P. Hutchinson, Hadwiger's conjecture and sixchromatic toroidal graphs, in: Graph Theory and Related Topics, Academic Press, New York, 1979, 35-40.

[4] Michael O. Albertson and Joan P. Hutchinson, Hadwiger's conjecture for graphs on the Klein bottle, Discrete Math. 29 (1980), 1-11.

[5] Michael O. Albertson and Joan P. Hutchinson, Extending colorings of locally planar graphs, J. Graph Theory 36 (2001), 105-116.

[6] Michael O. Albertson and Joan P. Hutchinson, Extending precolorings of subgraphs of locally planar graphs, (in preparation).

[7] Michael O. Albertson and Emily H. Moore, Extending graph colorings, J. Combinatorial Theory, Series B 77 (1999), 83-95.

[8] Michael O. Albertson and Emily H. Moore, Extending graph colorings using no extra colors, Discrete Math. 234 (2001), 125-132.

[9] Ken Appel and Wolfgang Haken, Every planar map is four-colorable, Bull. Amer. Math. Soc. 82 (1976), 711-712.

[10] G. A. Dirac, Map-colour theorems, Canadian J. Math. 4 (1952), 480-490.

[11] G. A. Dirac, Map colour theorems related to the Heawood color formula, J. London Math. Soc. 31 (1956), 460-471.

[12] G. A. Dirac, Map colour theorems related to the Heawood color formula (II), J. London Math. Soc. 32 (1957), 436-455.

[13] G. A. Dirac, Short proof of a map-colour theorem, Canadian J. Math. 9 (1957), $225-226$.

[14] Phillip Franklin, A six color problem, J. Math. Phys. 13 (1934), 363-369.

[15] H. Hadwiger, Über eine Klassifikation der Streckenkomplexe, Vierteljahrsch. Naturforsch. Ges. Zürich 88 (1943), 133-142.

[16] P. J. Heawood, Map-colour theorem, Quart. J. Pure Appl. Math. 24 (1890), 332-338.

[17] Tommy R. Jensen and Bjarne Toft, Graph Coloring Problems, John Wiley and Sons, Inc., New York, 1995.

[18] Bojan Mohar and Carsten Thomassen, Graphs on Surfaces, Johns Hopkins Univ. Press, Baltimore, 2001.

[19] Neil Robertson, Dan Sanders, Paul Seymour, and Robin Thomas, The four colour theorem, J. Combinatorial Theory, Series B 70 (1997), 2-44. 
[20] Neil Robertson, Paul Seymour, and Robin Thomas, Hadwiger's conjecture for $K_{6}$-free graphs, Combinatorica 13 (1993), 279-361.

[21] Carsten Thomassen, Color-critical graphs on a fixed surface, preprint, 1996.

[22] Carsten Thomassen, Color-critical graphs on a fixed surface, J. Combinatorial Theory, Series B, 70 (1997), 67-100.

[23] Carsten Thomassen, (personal communciation) 2002.

[24] Zsolt Tuza, Graph colorings with local constraints - a survey, Discuss. Math. Graph Theory 17 (1997), 161-228.

[25] K. Wagner, Über eine Eigenschaft der ebenen Komplexe, Math. Ann. 114 (1937), 570-590. 